\title{
A two-dimensional Purkinje eye tracker
}

\author{
MICHAEL R. CLARK \\ Stanford Research Institute, Menlo Park, California 94025
}

\begin{abstract}
The principle and operation of a two-dimensional eye tracker is described. The instrument makes use of the first and fourth Purkinje images to determine both eye position and direction of gaze. There is no physical contact with the eye. It is as accurate and as convenient to use as any previously used eye movement monitor.
\end{abstract}

This paper briefly describes a two-dimensional eye tracker that utilizes information on the spatial position of two eye reflections to determine eye position (see Cornsweet \& Crane, 1973). These two reflections, the first and fourth Purkinje images, move identically during eye translation and differently during eye rotation; thus, both translation and rotation can be determined separately. In utilizing this approach, one can combine the desirable features and eliminate most of the drawbacks found in other eye monitoring systems. The Purkinje Eye Tracker is as accurate as eye movement monitors which use contact lenses while being considerably more convenient; it is much more accurate than devices that utilize electrodes or eyeglass-mounted photoelectric techniques while being almost as convenient for many applications. Artifacts due to head or eye translations are minimized with this method.

When the human eye is illuminated with light (e.g., from a point source at optical infinity), there are four images, "Purkinje images," of this source created in the eye as shown in Figure 1. The first Purkinje image is a virtual image formed by reflection from the front surface of the cornea; the second image is almost coincident with the first and is created by light reflected from the rear of the cornea. The remaining light passes through the cornea and aqueous humor; a fraction of that light is reflected from the front surface of the lens and forms the third Purkinje image. This is a virtual image that is larger and more diffuse than the others; it is formed in a plane far removed from the plane of the other images. The fourth Purkinje image is created by light reflected from the back surface of the lens. This light forms a real image in the same plane (the plane of the eye pupil) as the much brighter first Purkinje image.

During eye translation, the first and fourth Purkinje images move together. During rotation, however, because the centers of curvature for these two "mirror" surfaces are not coincident, the two images move differentially. The resulting change in their spatial separation can be used to determine the pure angular rotation of the eye, since eye translations do not change the magnitude of separation. The Purkinje eye tracker effectively provides continuous electrical signal analogous to the horizontal and vertical components of the separation of these two images. This technique is

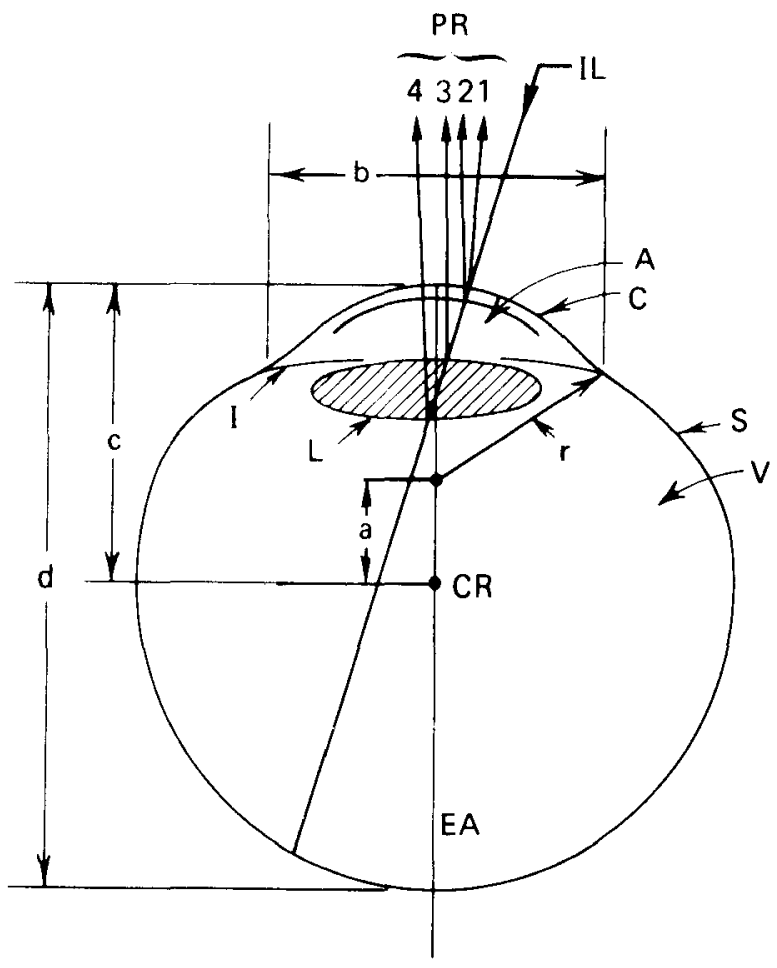

FIG. 1. Schematic diagram of the eye: $P R$, Purkinje reflections; IL, incoming light; $A$, aqueous; $C$, cornea; $S$, sclera; V, vitreous; $I$, iris; L, lens; CR, center of rotation; EA, eye axis; $a \approx 6 \mathrm{~mm}$, $b \approx 12.5 \mathrm{~mm}, c \approx 13.5 \mathrm{~mm}, d \approx 24 \mathrm{~mm}, r \approx 7.8 \mathrm{~mm}$.

ultimately limited by the quantum fluctuations in the very low-intensity fourth Purkinje image.

The positions of the first and fourth Purkinje images are depicted in Figure 2. The radius of curvature of the cornea is approximately $7.8 \mathrm{~mm}$. Thus, light from a distant point source would create a (virtual) first Purkinje image that lies in a plane $7.8 / 2$ or $3.9 \mathrm{~mm}$ from the front corneal surface. The (real) fourth Purkinje image is formed by reflection from the back of the lens. A single-mirror equivalent is shown by the dark line in Figure 2a that would form an identical image. This mirror has a radius of approximately $5.8 \mathrm{~mm}$ for relaxed accommodation and its center of curvature $\mathrm{C}_{4}$ is close to the corneal surface. The image planes for the first and fourth Purkinje images are almost exactly the same.

Assume, for purposes of explanation, that the equivalent mirror for the fourth Purkinje image has the 


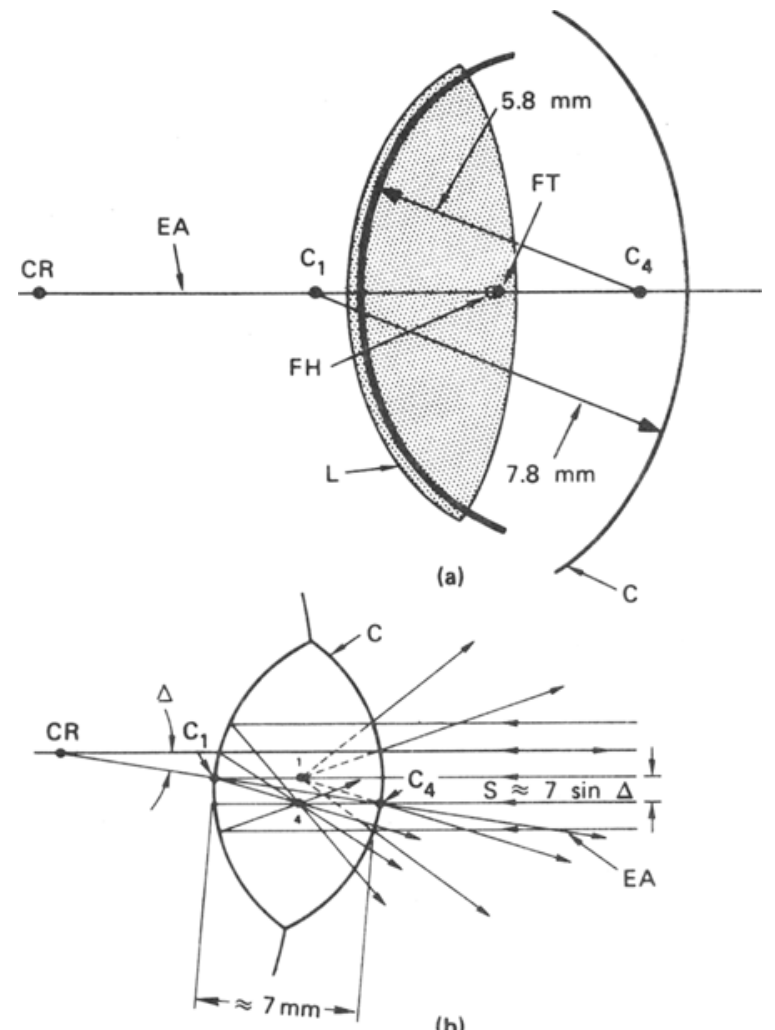

(b)

FIg. 2. Location of the first and fourth Purkinje images for (a) collimated light on the eye axis and (b) collimated light at angle $\Delta$ from optic axis of the eye: EA, eye axis; FT, first Purkinje image; FH, fourth Purkinje image; L, lens; C, cornea. The dark section of arc is the equivalent mirror for the fourth Purkinje reflection.

same curvature as the cornea, and they are separated by their radius of curvature as shown in Figure $2 b$. When the eye turns, the distance that each image moves is directly proportional to the distance from the center of rotation of the eye to the center of curvature of the surface that forms it. If the rotation is $\Delta$, the movement of the two images when viewed from the input axis is in the same direction with separation:

$$
\mathrm{S}=7 \sin \Delta \text { (millimeters) }
$$

With respect to the optic axis of the eye, however, the images move in opposite directions since one image is in front of its center of curvature and the other is behind its center of curvature. During translation, the images move through the same distance and in the same direction as the eye; thus, any change in separation is solely due to eye rotation.

An earlier version of the eye tracker system but one that is easier to describe is shown in Figure 3. The latest improvements are noted below. The light source (S), a $500-\mathrm{W}$ projection lamp, is imaged by Lens $\mathrm{L}_{1}$ onto the circular stop $\left(S_{1}\right)$. Stop $S_{1}$ is in the focal plane of Lens $L_{2}$ and is imaged by Lens $L_{3}$ onto the plane of the pupil of the eye. Stop $S_{2}$ is in the focal plane of Lens $L_{3}$ and therefore appears to the eye at optical infinity. The light is passed through a filter (F) with passband between .8 and 1.1 micrometer and is chopped at $1,200 \mathrm{~Hz}$ by a chopper wheel (CW).

Light from the Purkinje images is reflected by a dichroic mirror (DC), imaged by Lens $L_{4}$, reflected by Mirror $M$ and divided by a beam splitter (BS). Signals $M_{x}$ and $\mathrm{M}_{\mathrm{y}}$, derived from the quadrant photodetector $\left(\mathrm{P}_{1}\right)$ as shown in Figure 4, are used to drive two motors that move the mirror $(\mathrm{M})$ horizontally and vertically so as to stabilize the position of the first Purkinje image on $\mathrm{P}_{1}$. The position of Photocell $P_{4}$ is controlled by Signals $P_{x}$ and $\mathrm{P}_{\mathrm{y}}$ which drive two additional motors to center the photocell on the fourth Purkinje image. Thus, the signals $P_{x}$ and $P_{y}$ are directly related to eye rotation, since it represents movement of the fourth Purkinje image with respect to the first Purkinje image. The mirror (M)



FIG. 3. Schematic of the eye-tracker optical system: VT, visual target; $\mathrm{R}$, allowed range of eye movements; IA, input axis; $\mathrm{CA}$, collecting axis; $\mathrm{CA}^{\prime}$, extension of collecting axis; $\mathrm{S}$, light source; $\mathrm{S}_{\mathrm{I}}$, artificial pupil imaged at pupil of eye; $\mathrm{CW}$, chopper wheel; $\mathrm{S}_{2}$, source of Purkinje pattern, imaged at infinity; $\mathrm{DC}$, dichroic mirror; $\mathbf{M}$, front surface mirror; $\mathbf{M}_{x}$ and $\mathbf{M}_{y}$, motors that drive $\mathbf{M}$ in $x$ and $y$ direction, respectively; $\mathrm{BS}$, beam splitter; $\mathrm{P}_{1}$ and $\mathrm{P}_{4}$, quadrant photocells; $A_{4}$, aperture in front of $P_{4}$. Focal lengths of lenses $L_{2}, L_{3}$, and $L_{4}$ are 60,150 , and $90 \mathrm{~mm}$. 

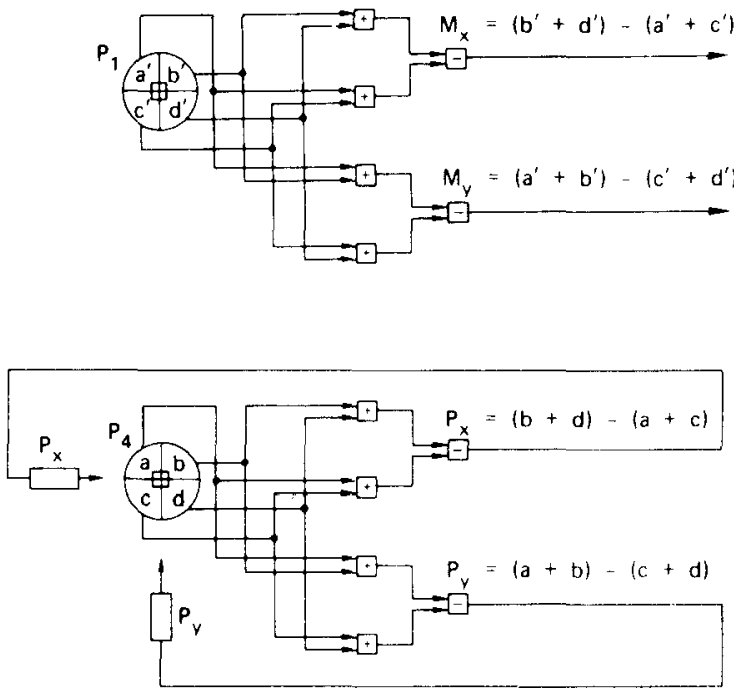

Fig. 4. Photocell connections: $\mathrm{M}_{x}$ and $\mathrm{M}_{y}$, signals that drive the two-dimensional mirror in $x$ and $y, P_{x}$ and $P_{y}$, motors that drive $\mathrm{P}_{\mathrm{A}}$ in $x$ and $y$ directions.

removes any common-mode (i.e., identical) movements of the two images, e.g., due to eye translation. The four motors are arranged in a feedback loop as shown in Figure 5. The motion sensor (MS) is accurate to 1 micrometer and the drive circuits are flat to $100 \mathrm{~Hz}$.

The subject is positioned by a forehead-chin rest combination or a bite bar and sees the target through the dichroic mirror (DC). The angle between the eye axis and input axis is labeled $\Delta$ in Figure 3 . As the eye rotates toward Position $\mathrm{A}$, the angle $\Delta$ decreases, and the separation of the first and fourth Purkinje images decreases. Eventually, light from the first image enters Photocell $\mathrm{P}_{4}$ and prevents any further eye tracking. As $\Delta$ increases, a situation is reached such that the pupil of the eye occludes the fourth Purkinje image; again tracking is prevented. The range of this eye tracker is a viewing field approximately $20 \mathrm{deg}$ in diam. (The latest version has a range of $30 \mathrm{deg}$ ).

Blinks are detected by monitoring the sum of all four quadrant signals from $\mathbf{P}_{1}$. This voltage is nominally constant except during a blink when the eye tracker holds the last value of eye position until the termination of the blink.

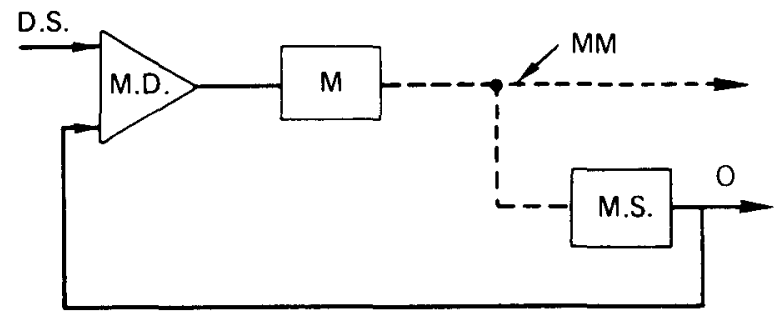

FIG. 5. Feedback circuit around each motor: D.S., drive signal; M.D., motor driver; M, motor; MM, mechanical motion; M.S., motion sensor, Hewlett-Packard DCDT displacement transducer; $O$, output signal.
There have been several improvements in subsequent eye trackers. These changes are summarized below.

The light source (S), chopping wheel (CW), and filter (F) have all been replaced with an infrared LED that is electronically chopped. The input axis (IA) may be adjusted to one of three positions corresponding to narrow-, normal-, or wide-angle viewing.

Tolerance of head movement-at least sufficient tolerance to eliminate the need for a bite bar-has universally been considered a desirable feature. The early trackers could be used with a chin and forehead rest, but without much margin to spare. The recent ones have been designed to allow $\pm .5 \mathrm{~cm}$ head movement in all three axes. To accomplish this, automatic focusing was added to permit movement of the head along the optic axis. It was expected that this design change would provide the additional benefit of eliminating focus adjustment from the manual alignment procedure.

The early trackers illuminated not only the pupil but also a large portion of the iris. This was necessary to allow lateral translation of the eye without the eye moving out of the illuminating beam. Illumination of a larger area than the pupil causes two problems. First, more infrared energy than is absolutely necessary is projected into the eye, thus reducing the maximum safe light level. Second, light reflected from the iris degrades the tracker performance, because it tends to spill over onto the Purkinje images via lens aberrations and because it causes spurious quadrant photocell outputs.

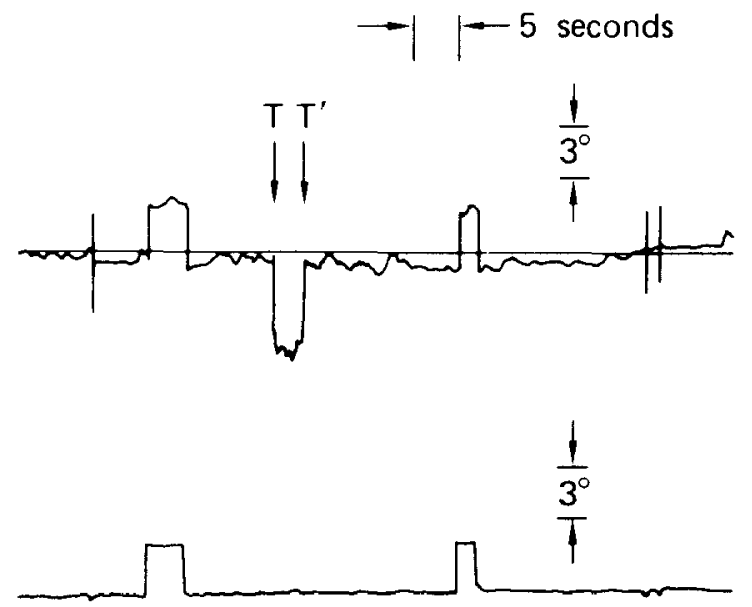

4

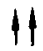

FIc. 6. Upper, horizontal eye movements recorded from firstPurkinje-image tracker; lower, horizontal eye movements recorded from fourth-Purkinje-image tracker. The upper trace shows the wandering baseline, typical of corneal image trackers. Both traces record two $3^{\circ}$ horizontal eye movements. During the interval $T$ - $\mathrm{T}^{\prime}$, the subject's head was translated $0.5 \mathrm{~mm}$ horizontally, which shows in the upper trace but not the lower. Arrows at the bottom indicate eye blinks. 


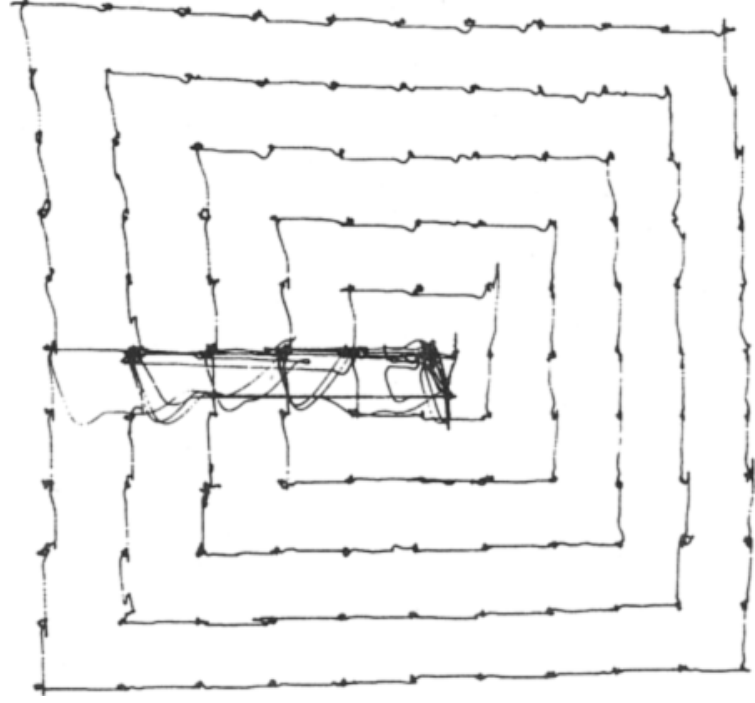

Fic. 7. An $x, y$ recording of eye movements (movements of $P_{4}$ ) over an $11 \times 11$ array of illuminated points. Adjacent points were separated by $1.8^{\circ}$. The ragged traces on the horizontal axis, left of center, are due to eye blinks.

The new trackers steer the illuminating beam so that it always falls within the pupil. It is expected that the field over which the gaze can be tracked will also be improved by this modification, which will allow coverage of $\pm 15 \mathrm{deg}$ in both horizontal and vertical directions.

Tracking of the first Purkinje image is accomplished by rotating a mirror that lies in the optical path from the eye to the photodetectors. In the older trackers, the optical path was reflected from the mirror (M) through a right angle, as shown in Figure 3. This introduced a predictable but annoying distortion of the eye tracker output vs. gaze direction. In the newer trackers, this distortion has been eliminated (to a first order of approximation) by reflecting the optical path back upon itself with this mirror

The tracking of the fourth Purkinje image is now achieved by moving an additional mirror in a manner completely analogous to the operation of Mirror $M$ instead of moving the Photocell $P_{4}$.

Figure 6 demonstrates the ability of this instrument to measure pure eye rotation without contamination due to translation. The upper trace is typical of records from corneal eye trackers with the normal wandering of the baseline due to translation, and an induced translation in Period TT $^{\mathbf{l}}$. The lower trace demonstrates the absence of eye translation effects in the eye rotation responses. Figure 7 shows typical results in an 18-deg field; Figure 8 depicts a variety of eye fixations and movements. These devices are capable of measuring eye movements with an error of about $1 \mathrm{~min}$ of arc. Mechanical elements in the tracker have a 4-msec lag in response to a constant velocity input. This is analogous to a lag of $1.2 \mathrm{deg}$ in a $5-\mathrm{deg}$ saccade with a velocity of

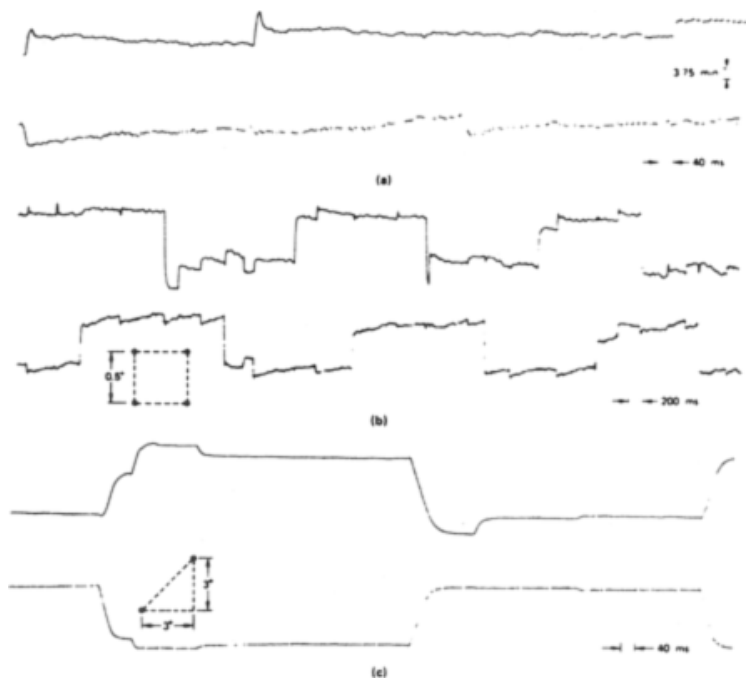

Fic. 8. (a) Fixation record; (b) repetitive traverse of the corners of a $1^{\circ}$ square; (c) diagonal saccadic movement with $3^{\circ}$ horizontal and vertical components. Upper, horizontal movements; lower, vertical movements.

$300 \mathrm{deg} / \mathrm{sec}$. Smaller saccades have corresponding smaller lags. The response of the servo systems is being improved to reduce this error.

Work is presently being done to add the Cornsweet-Crane optometer (Cornsweet \& Crane, 1970), for measuring the refractive power of the eye, to the Purkinje eye tracker. This composite system will be capable of measuring all of the essential visual focusing and tracking mechanisms of the eye (including pupil size) and therefore could become an invaluable research tool.

This instrument will shortly be used in a variety of ways within our own laboratory. Kelly and Savoie (1973) will utilize the eye tracker to stabilize spatio-temporal grating patterns to isolate the effects of eye movements on threshold measurements. Cooper (1974) plans to study the interrelationships of speech and vision. Cornsweet and Crane (1970) and Clark (1974) also intend to use an eye tracker optometer instrument to measure the relationships between eye movement and accommodation. All of these efforts will utilize a computer for on-line control of the experiments and analysis of the results.

In summary, a two-dimensional eye tracker that is both accurate and easy to use is described. It effectively monitors the changes in separation of the first and fourth Purkinje images to provide an indication of eye rotation undisturbed by eye translation. It operates with light in the near infrared range which is invisible to the subject. A standard chin-forehead rest is adequate for alignment of the subject. It takes only a few minutes to align each new subject. 


\section{REFERENCES}

Clark, M. R., \& Stark, L.. Control of human eye movements: III. Dynamic characteristics of the eye tracking mechanism. Mathematical Biosciences, 1974, 20, 239-266.

Cooper, $R$. The control of eye fixation by the meaning of spoken language. Cognitive Psychology, 1974, 6, 84-107.

Comsweet, ' 1 '. N., \& Crane, H. D., Servo-controlled infrared optometer. Journal of the Optical Society of America, 1970, 60, 548-554.

Cornsweet, ' $\Gamma$. N., \& Crane, H. D. Accurate two-dimensional eye tracker using first and fourth Purkinje images. Journal of the Optical Society of America, 1973,63,921-930.

Kelley, D. H., \& Savoie, R. E. A study of sine-wave contrast sensitivity by two psychophysical methods. Perception \& Psych ophysics, 1973. 14, 313-318. 